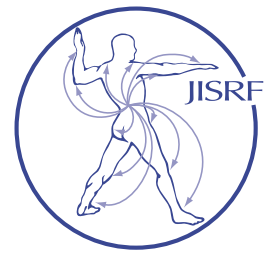

\title{
Bio-Occlusive Gauze with Tegaderm: A Dressing for Surgical Wounds in Primary THA and TKA
}

\author{
Chowdhry $M^{1}$, Dipane $M^{1}$, McPherson $E^{1}$
}

\section{Abstract}

Background: We introduce a simple, cost-effective bioocclusive dressing to be used for primary total hip arthroplasty (THA) and primary total knee arthroplasty (TKA).

Methods: The gauze-Tegaderm ${ }^{\mathrm{TM}}$ (GT) dressing consists of a $5 \mathrm{~cm}$ wide 8-layered gauze covered by 3 to 5 mediumsized Tegaderm transparent films. We prospectively evaluated 100 consecutive primary THA's and 107 consecutive primary TKA's utilizing this dressing with a minimum of one-year follow-up.

Results: In the primary THA group, there was one surgical site infection (SSI) requiring oral antibiotic treatment. There were no cases of periprosthetic joint infection (PJI). In the primary TKA group, there were two surgical site infections requiring oral antibiotic treatment and one case of chronic PJI requiring a two-stage exchange protocol.

Discussion: Our SSI and PJI rates are comparable to published rates in the literature. The GT dressing is a simple, inexpensive dressing that can compete against the many proprietary bio-occlusive dressings that are more expensive and are not readily available worldwide. Our favorable review has merited a large volume randomized controlled study comparing the GT dressing to another proprietary bioocclusive dressing.

\section{Background}

As the world population continues to rise, so does the prevalence of degenerative joint disease. Currently, it is estimated that more than 2 million total hip arthroplasty (THA) and total knee arthroplasty (TKA) procedures are performed worldwide $[1,2]$. Although these total joint arthroplasty (TJA) procedures are very successful, periprosthetic joint infection (PJI) is a major complication that occurs at a steady rate worldwide. The combined PJI rate for primary THA and TKA procedures is estimated to be between 1-6\% $[\underline{3}, \underline{4}]$. This is a major challenge to all healthcare institutions and personnel, as the cure requires an inordinate amount of time and consumes a significant portion of one's healthcare budget. As a result, in the last decade, PJI prevention has been emphasized by governmental and healthcare organizations. Methods to reduce PJI include preoperative optimization of the patient's health, pre-admission skin cleansing, and adherence to strict intra-operative measures to reduce joint implant microbial colonization. Additionally, post-operative wound care measures have been highlighted to reduce the rate of local surgical site infections (SSI) that can progress into a PJI. Consequently, the healthcare market has seen a proliferation of various wound dressings as a means to reduce SSI.

The aim of any post-operative wound dressing is to absorb wound blood and exudate while reducing local bacterial load to the surgical site. Furthermore, the dressing should

Keywords: Postoperative, Dressing, Bio-Occlusive, THA, TKA,

TJA, Gauze, Tegaderm, Primary, Arthroplasty, SSI, PJI

Level of Evidence: AAOS Therapeutic Level IV

Educational Value \& Significance: JISRF Level A 
keep the environment around the wound moist enough to prevent desiccation and accelerate natural wound healing [5]. Many companies have developed bactericidal/bacteriostatic dressing coverings to mitigate SSI. All advertised dressings report effective reduction of SSI to some degree, but the costs of such dressings are relatively expensive. With the costs of healthcare rising throughout the developed world, all healthcare personnel are cognizant of providing effective treatment at lower costs. This applies to all aspects of perioperative total joint arthroplasty (TJA) care, including perioperative dressings.

In this review, we introduce a simplified surgical dressing that we believe provides effective treatment of perioperative TJA wounds. The design consists of an 8-layered simple gauze dressing covered with an occlusive polyurethane film (Tegaderm $^{\mathrm{TM}}, 3 \mathrm{M}$, St. Paul, MN). It is simple, readily available, and economical. The gauze dressing over the wound acts as a highly absorbent pad to absorb any excess exudate as well as keeping the immediate surroundings moist. The occlusive polyurethane film (Tegaderm), applied over the gauze, provides a waterproof seal to the wound. It still allows for the exchange of water vapor while inhibiting the entry of bacteria. This keeps the wound moist as well as free from any external contaminate []. It serves as a significantly cheaper alternative to its counterpart dressings currently available on the market. To date, to the best of our knowledge, no study has shown the effectiveness of this particular dressing combination in terms of prevention of SSI and PJI, nor the calculated reduction in the cost for the healthcare system. The objective of this study was to evaluate the effect of using this dressing combination on the occurrence of PJI and SSI. We compare our results to the reported rates in the literature. In addition, we assess the financial impact of utilizing this simple perioperative dressing. We hypothesize that the Gauze-Tegaderm dressing combination will be as effective as other "modern" dressings discussed in the literature while providing a significant cost savings.

\section{Methods}

Between January 2015 and December 2016, 796 TJA procedures were performed at our single TJA quaternary referral institution by the senior author (ejm). The TJA procedures included total shoulder arthroplasty (TSA), total hip arthroplasty (THA), and total knee arthroplasty (TKA). During this time period there were 395 revision TJA procedures, 115 resection TJA procedures, 52 reimplant TJA procedures, and 234 primary TJA procedures. We selected our primary THA and primary TKA procedures as the basis for this study. Beginning January 2015, we started the pro- spective study in which we covered all consecutive primary THA and TKA procedures with a gauze-tegaderm dressing combination. We selected a minimum follow-up period of one year for this report.

The constituents of the gauze-Tegaderm (GT) surgical dressing are sterile $4 \times 4$ inch gauze dressing pads (Medline, Mundelein, IL) and $4 \times 4.75$ inch Tegaderm $^{\mathrm{TM}}$ Film covers. The technique of assembling and applying the GT dressing was the same for THA and TKA procedures; this technique remained constant over the entirety of the study period. The dressing assembly required unfolding 4 sterile gauze dressings and laying them on top of one another. Next, the 4 layers were folded in half to a width of 2 inches $(5.08 \mathrm{~cm})$. The now 8-layered gauze was applied over the surgical site and any excess at the ends was cut off. The gauze was then covered with the Tegaderm films. The films were overlapped approximately $1 \mathrm{~cm}$ to provide an impervious seal of the surgical incision. They were applied in a fashion to have at least $2 \mathrm{~cm}$ of skin contact circumferentially around the gauze dressing. For THA procedures, the GT dressing was applied at the termination of the surgical procedure with the patient in the lateral decubitus position. Prior to the application of the dressing, the skin was cleaned with sterile saline solution via a laparotomy sponge (Medline, Mundelein, IL) and completely dried with a dry laparotomy sponge. The Tegaderm was applied over the gauze and gently pushed onto the skin. We were strict not to stretch the Tegaderm during application in the interest of preventing skin blistering. For TKA procedures, the GT dressing was applied at the termination of the surgical procedure with the knee flexed at $90^{\circ}$. The skin was cleaned and dried in a similar fashion to the THA application. Again, the Tegaderm was gently pushed digitally onto the skin avoiding any stretching of the cover. For all primary TKA procedures we used a joint drain that was exited over the lateral mid-thigh. The drain was secured with a smaller $4 \times 3 \mathrm{~cm}$ GT dressing. The GT dressing applications are illustrated in Figures 1a-1c.

Dressing changes were performed on the surgical floor when blood or serous fluid extended to the edge of the gauze. If the surgical dressing required a change, a similar dressing was reapplied after cleaning the surgical site with alcohol pads and/or sterile dry gauze. If the surgical dressing remained dry and intact, the patient was discharged with instructions to remove the dressing on post-operative day 7 or 8. Patients were allowed to shower with the waterproof GT dressing. Similarly, if the dressing was changed, the patient was discharged with the last GT dressing and instructed to remove the dressing on post-operative day 7 or 8 .

All THA procedures were performed using a less invasive posterolateral incision [7]. The patient was positioned and secured in the lateral decubitus position utilizing the Hip 

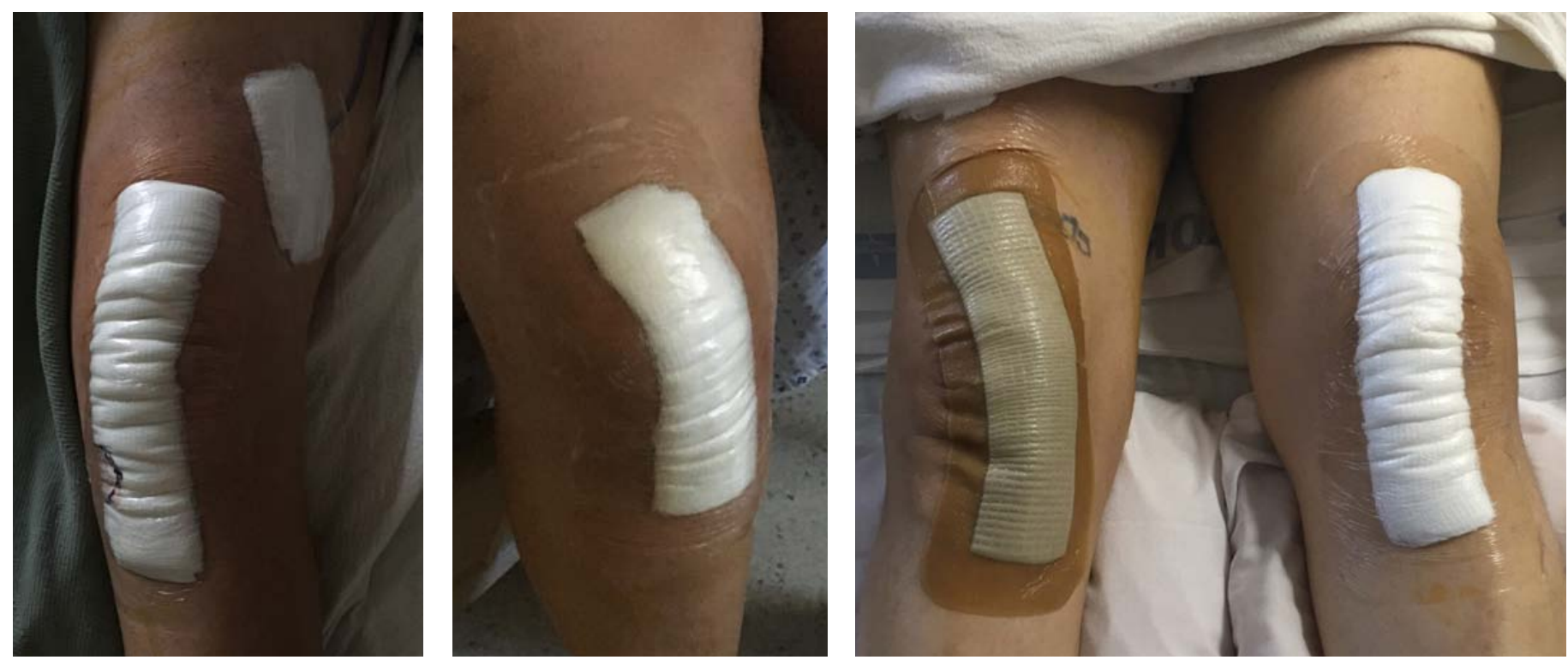

Figure 1(a-c): Photographs demonstrating application of Gauze-Tegaderm (GT) dressing in Primary TKA cases.

Grip System (SunMedica, Redding, USA). The entire limb, hip, and pelvis were first cleansed and wiped with 70\% isopropyl alcohol wipes (McKesson, Santa Fe Springs, USA) and allowed to dry. The entire limb, hip, and pelvis were treated with DuraPrep ${ }^{\mathrm{TM}}(3 \mathrm{M}$, St. Paul, USA) and draped sterilely with disposable paper drapes. Exposed skin surfaces were covered with an $\operatorname{Ioban}^{\mathrm{TM}}$ dressing cover $(3 \mathrm{M}$, St. Paul, USA) that was removed at the termination of skin closure. A first generation cephalosporin (Ancef, Baxter International, Deerfield, USA) was administered intravenously 30 minutes prior to incision and continued for 24 hours. If a patient stated an allergy to penicillin, a test dose of Ancef was administered and, if after 15 minutes there was no observable reaction, IV Ancef was continued. If the patient had a known or documented allergy to Ancef, IV 1 gram Vancomycin was administered prior to incision and was continued for 24 hours. Throughout the procedure, the tissues were injected with a periarticular joint cocktail for pain management. The pain block cocktail is listed in Table 1. The tissues were strategically injected with a multi-stab technique with a 23 gauge needle [ $\underline{8}$ ].

The hip incision was made long enough to allow for comfortable access and exposure to the hip. A cementless acetabular cup was used in all cases. A titanium, porous plasma spray hemisphere cup was inserted (Magnum or Ranawat Burstein, Biomet, Warsaw, USA) with a press-fit technique of a $1 \mathrm{~mm}$ underream. Just prior to implant insertion, the acetabular bone was hand lavaged with 100 to $150 \mathrm{cc}$ of sterile saline solution containing 1 gram of Bacitracin (APP Pharmaceuticals, Schaumburg, USA) mixed in one liter of sterile saline solution. For the femoral stem, a cementless stem was used in all cases (TaperLoc, Biomet, Warsaw, USA). This was a titanium alloy, proximal, porous plasma spray ta-
Figure 1a.64-year-old male on post-operative day one. The GT dressing covers the knee incision and drain site. Notice the blood stain on the inferior part of the gauze (highlighted in black marker). The transparent Tegaderm allows visualization of the gauze dressing underneath. The dressing is changed when the underlying gauze becomes stained from edge to edge with fluid and/or blood.

Figure 1b.70-year-old female on post-operative day two. The GT dressing on the drain site has been removed. The GT dressing completely allows knee flexion to 90 degrees without irritating the skin. This patient went home with this dressing, which was removed by the patient on post-operative day seven.

Figure 1c. 68-year-old male with staged primary TKAs one week apart. The GT dressing was applied on the initial TKA (left), seen on post-operative day 8. For demonstration, we applied the bio-occlusive Aquacel dressing on the contralateral knee, seen on post-operative day two. Note how the Aquacel dressing pulls upon the lateral skin. This type of pulling force can cause skin blisters with repetitive knee range.

pered stem. The femoral canal was prepared by serial broach technique utilizing a $0.75 \mathrm{~mm}$ undersized press-fit at stem insertion. Prior to stem implant insertion, the femoral canal was lavaged with 100 to $150 \mathrm{cc}$ of sterile saline solution containing Bacitracin. The acetabular and femoral stem implants were inserted using a "no touch" technique as much as possible. Prior to closure the entire wound was hand la-

Table 1. Periarticular Pain Block Cocktail (Primary TKA \& THA)

\begin{tabular}{|c|}
\hline $\begin{array}{c}\text { 20cc Bupivacaine Liposome (Exparel } \mathrm{Q}) \\
+\end{array}$ \\
\hline 1cc Methylprednisolone Acetate \\
+ \\
\hline 2cc Ketorolac Tromethamine \\
+ \\
\hline 25cc Bupivacaine HCI with Epinephrine $(5 \mathrm{mg} / \mathrm{mL})$ \\
\hline Total Volume $=48 \mathrm{cc}$ \\
Not Diluted with Sterile Saline \\
\hline
\end{tabular}


vaged using a 25cc Asepto syringe (McKesson, San Francisco, CA) with 200 to $250 \mathrm{cc}$ of sterile saline solution containing Bacitracin. The top surgical gloves were changed at the beginning of closure (double glove technique was employed for all surgical personnel). A multilayered closure was performed using all absorbable sutures. Number One Vicryl and 2-0 Vicryl (Ethicon, Somerville, NJ) sutures without antibiotic coating were used for all layers. The skin was closed with a subcuticular technique using 3-0 Monocryl (Ethicon, Somerville, USA). The skin was reinforced with $1 / 2$ inch steristrips (3M, St. Paul, USA) cut to a width of $2.5 \mathrm{~cm}$ so that they would be covered by the GT dressing. The steristrips were applied with a thin application of Benzoin $(3 \mathrm{M}$, St. Paul, USA) applied only to a width of $2.5 \mathrm{~cm}$ of the skin.

All TKA procedures were performed using a less invasive paramedial incision with a medial parapatellar arthrotomy [9]. The knee and limb were secured utilizing the Knee Grip System (SunMedica, Redding, USA). The entire limb was initially cleansed with alcohol wipes and allowed to dry. A pneumatic tourniquet was applied into the most proximal thigh. The tourniquet pressure was $275 \mathrm{~mm} / \mathrm{Hg}$ in all cases. The tourniquet was inflated prior to skin incision and deflated after cementing of the implants. The entire limb was treated with Duraprep and draped sterilely with disposable paper drapes. Exposed skin surfaces were covered with an ioban dressing cover. The ioban was removed at the termination of skin closure. Intravenous antibiotics were administered using the same protocol as the THA procedures. Additionally, the same periarticular pain block cocktail was injected into the knee tissues. For all TKA procedures, an adductor block using $20 \mathrm{cc}$ of $0.5 \%$ Ropivacaine was administered prior to the surgical procedure.

The knee incision was made long enough to allow for comfortable access and exposure to the knee. The Vanguard Total Knee System ${ }^{\mathrm{TM}}$ (Biomet, Warsaw, USA) was used in all cases. An anterior stabilized Vitamin E reinforced polyethylene bearing was used in all cases except when a constrained knee system was required for severe deformities. All patellae were resurfaced with a polyethylene 3-peg dome. All implants were cemented with Palacos Cement (Biomet, Warsaw, USA) without antibiotics added to the PMMA powder. Prior to cementing of the implants, all boney surfaces of the knee were pulse mechanical lavaged with sterile saline solution containing Bacitracin. Top gloves were changed for insertion of implants and also changed at the time of closure of the knee. Just prior to closure, the knee was lavaged with 1 liter pulsed mechanical lavage using sterile saline solution containing Bacitracin. All layers of the knee incision were closed at $90^{\circ}$ of flexion, including the subcuticular layer. A 10 French Blake wicking silicone drain (Ethicon, Somerville, USA) was placed into the lateral gutter of the knee and brought out of the skin at the anterolateral mid-thigh. The drain was removed on the first postoperative day. A multilayer closure was performed using all absorbable sutures without antibiotic coating. The arthrotomy was closed with number 1 and 2-0 Vicryl sutures. The subcutaneous layers were closed with 2-0 and 3-0 Vicryl sutures and the subcuticular layer was closed with a subcuticular technique using 3-0 Monocryl sutures. The skin was reinforced with $1 / 2$ " steristrips cut to a width of $2.5 \mathrm{~cm}$ and applied with a thin coat of Benzoin. The skin was cleaned and dried prior to application of the steristrips, after which the GT dressing was applied.

All THA and TKA procedures were performed with body exhaust suits (Flyte, Stryker, Kalamazoo, USA) in non-laminar flow dedicated total joint rooms. Anesthesia consisted of a general anesthetic combined with a spinal anesthetic. Intrathecal morphine sulfate was not used in any cases. Patients were started in physical therapy within 6 hours of the procedure with standing and walking. For thromboembolic prophylaxis, a graduated risk assessment protocol was utilized by the medical team. The default, low risk, patients were treated with mechanical foot pumps and enteric coated aspirin $(325 \mathrm{mg})$ daily. Higher risk patients were treated with other antiplatelet inhibitors or oral warfarin with a target INR of 2.8 to 3.0. On rare occasion, the very high-risk patients were treated with a pre-operative removable inferior vena cava filter, which was removed 3-4 months after the joint replacement procedure.

Preoperatively, all patients were scored for periprosthetic joint infection risk using the Musculoskeletal Infection Society (MSIS) risk scoring system, calculating both a systemic host grade (A, B, or C) and a local extremity grade $(1,2$, or 3$)[10,11]$. All patients were followed routinely at 6 weeks, 12 weeks, and yearly thereafter. Additional treatment was provided as needed. All complications or additional surgeries were documented. All clinical follow-up was with the operating surgeon. TKA procedures were evaluated with radiographs, Knee Society Scoring and Oxford Scoring at regularly defined intervals. THA procedures were evaluated with radiographs, Hip Society Scoring, and Oxford scoring at regularly defined intervals. When there was any suspicion of a PJI, the patient was assessed with serum blood testing. This included Complete Blood Count (CBC), quantitative c-reactive protein levels, and an erythrocyte sedimentation rate (ESR). When indicated, all joint aspirations were performed by the operating surgeon. All cultures were sent for a 14-day bacterial growth protocol. Fungal and mycobacterial plates were reviewed for a 6-week duration. A PJI was defined using the major and minor criteria as set forth by the International Consensus on Periprosthetic Joint Infection [12]. 


\section{Results}

In this study there were 100 primary THA procedures in 91 patients and 107 primary TKA procedures in 100 patients. For the THA group, there were 48 females and 52 males. The average age was 72 (range 51-98). Average body mass index (BMI) was 27 (range 14-46). The main diagnosis for needing the THA procedure was primary osteoarthritis in 48 patients, developmental dysplasia (DDH) in 32 patients, acute femoral neck fracture with joint arthritis in 9 patients, rheumatoid arthritis in 5 patients, avascular necrosis in 3 patients, and acetabular fracture in 3 patients. The MSIS scores for the study group consisted of 51 A Hosts, 42 B Hosts, and 7 C hosts. Ninety-one patients had a Type 1 limb score (local extremity score), while 9 patients had a Type 2 limb score. Operative blood loss was measured and averaged 255cc (range 50-500). Four patients required a post-operative blood transfusion. The average incision length was $11.8 \mathrm{~cm}$ (range 9 to 15). The average number of Tegaderm films used was 3.4 (range 3-5). The GT dressing was changed $44 \%(\mathrm{~N}=44)$ of the time prior to discharge. Table 2 displays the calculated total costs of the THA dressing application and compares this to an estimated cost of a silver-impregnated occlusive wound dressing (10-inch Aquacel $^{\mathrm{TM}}$, ConvaTec, Deeside, UK) that is available at our institution. At latest follow-up, an average of 18.1 months (range 12.9 to 24), there were no cases of PJI. No patients required additional surgery for an SSI or wound drainage. Two patients were prescribed oral antibiotics at their 6-week post-op evaluation for redness surrounding a localized suture reaction (i.e., "split sutures"). There were 3 reoperations performed. One patient dislocated at 3 weeks post-operatively, requiring an open reduction and revision of the acetabular cup. One patient underwent a removal of heterotopic bone at 10 months for symptomatic pain with hip flexion limited to $80^{\circ}$. One patient required revision at one week due to peri-prosthetic fracture of the femur. Other compli-

Table 2. Calculated Costs of GT Dressing Supplies with Comparison to Estimated Aquacel Costs

\begin{tabular}{|l|c|c|c|c|}
\hline & $\begin{array}{c}\text { Total \# } \\
\text { Dressing } \\
\text { Applications } \\
\text { Hip }\end{array}$ & $\begin{array}{c}\text { Calculated } \\
\text { Costs*_ } \\
\text { Hip (USD) }\end{array}$ & $\begin{array}{c}\text { Total \# } \\
\text { Dressing } \\
\text { Applications } \\
\text { Knee }\end{array}$ & $\begin{array}{c}\text { Calculated } \\
\text { Costs*_ } \\
\text { Knee (USD) }\end{array}$ \\
\hline GT Dressing & 144 & $\$ 432.00$ & 155 & $\$ 465.00$ \\
\hline $\begin{array}{l}\text { Estimated } \\
\text { Comparable } \\
\text { Aquacel } \\
\text { Dressing }\end{array}$ & 144 & $\$ 5,332.32$ & 155 & $\$ 5,739.65$ \\
\hline
\end{tabular}

*At our institution the acquisition cost is \$0.08 (USD) for one 4" 44 " gauze sponge pack (10 sponges) and \$0.59 (USD) for one Tegaderm film cover. A comparable Aquacel 3.5” x10" dressing cover costs $\$ 37.03$ (USD). cations were encountered that did not necessitate reoperation. One patient suffered from bilateral DVT at 12 weeks post-operatively. Another patient had a partial femoral nerve palsy with post-operative quadriceps power as $3 / 5$. This fully recovered. Lastly, one patient had a non-displaced greater trochanteric fracture intra-operatively that did not require any further intervention.

For the TKA group, there were 66 females and 41 males. The average age was 71 years (range 33 to 89). Average body mass index (BMI) was 26 (range 16-47). The main diagnosis for needing the TKA procedure was osteoarthritis in 90 patients, rheumatoid arthritis in 12 patients, and post-traumatic in 5 patients. For MSIS scoring, there were 54 A Hosts, 48 B Hosts, and 5 C Hosts. Eighty-seven patients had a Type 1 limb score (local extremity score), while 20 patients had a Type 2 limb score. The average measured intraoperative blood loss was 95cc (range 35-400). Only 1 patient required 1 unit of fresh frozen plasma preoperatively for known coagulopathy and cirrhosis. The average incision length was $12.4 \mathrm{~cm}$ (range 10-16). The average number of Tegaderm films used was 5.3 (range 5-7). The GT dressing was changed $45 \%(\mathrm{~N}=48)$ of the time prior to discharge. Table 2 displays the calculated total costs of the TKA dressing application and compares this to an estimated cost of the comparable Aquacel dressing. At latest follow-up, an average of 17.2 months (range 12.1 to 24), there was 1 case of PJI. This patient was successfully treated with a 2-stage revision arthroplasty. No other patients required additional surgery for SSI or wound drainage. Two patients were prescribed oral antibiotics at their 6-week postoperative evaluation for redness surrounding a localized suture reaction (i.e., split sutures). One patient also suffered from a loose tibial component 8 months postoperatively, requiring revision arthroplasty. Among complications not requiring reoperation, 4 patients developed joint arthrofibrosis requiring subsequent manipulation of the replaced knee joint, 1 patient suffered from a foot drop and fully recovered at 4 months, 1 patient had a DVT at 8 weeks, and 1 patient suffered from a superficial wound dehiscence requiring a wound vac. This was a patient with rheumatoid arthritis who went onto complete healing.

\section{Discussion}

Reduction of perioperative infection after total joint arthroplasty (TJA) is of paramount importance as infection is one of the most potentially disastrous complications that can occur. Superficial surgical site infection (SSI) can progress and result in deep periprosthetic joint infection (PJI). A PJI has enormous 
consequences, not only to the patient, but also to the healthcare community at large. Typically, a PJI requires reoperation to clear the infection and, if the acute PJI is not resolved, the implants require removal in either a single-stage or two-stage protocol. The costs of treating a chronic PJI could well pay for a further 10-30 primary TJA procedures.

Primary TJA wounds are classified as "clean," acute wounds with only moderate exudation [13]. The wound exudate is rich in IL-1, PDGF, EGF, and TGF-beta, all of which modulate connective tissue formation and epidermal migration [14]. Winter's research has demonstrated that a moist microenvironment enhances the wound healing process [15]. However, in some instances, some wounds can be highly exudative with persistent leakage. Ironically, this excess fluid could act as the breeding ground for microorganisms and cause infection. Thus, the ideal wound dressing should be able to absorb any excess exudate, but provide a moist microenvironment for optimal wound repair [16].

A unique challenge for the THA/TKA wound dressing is its direct application over a moving joint. The dressing must allow for functional range of motion, often over fragile elderly skin, without causing significant skin friction, shearing, and/or blistering. In addition, primary TJA is often associated with postoperative soft tissue edema, whereby there can be a substantial increase in skin circumference. Thus, a dressing must accommodate daily fluctuating skin circumference changes without causing significant skin friction and/or shearing. Any dressing that increases skin shear forces, increases the risk for blister formation. Blistering leads to breaks in the skin protective barrier and increases the risk of SSI []․ Therefore, an ideal dressing should be flexible with range of motion and must accommodate cyclic fluctuations in periarticular joint circumference. Lastly, Odland's research demonstrated that blisters heal faster if left unbroken [17]. Hence, a dressing with mechanical properties that limit blister formation and rupture would be ideal.

Cost conscious comprehensive medical care has become the normative process, competing against advancing medical technology and parabolic escalations in healthcare costs [18]. All aspects of orthopaedic surgical care are now carefully scrutinized with the advent of comprehensive medial informatics. Informatics programs allow comparisons of treatments between surgeons, OR teams, hospitals, and healthcare systems; providing effective safe treatment at reduced costs is the goal. The treating surgeon, going forward, will have to adapt to these changes and must take a leadership role in determining strategic changes in healthcare delivery that considers cost and benefit to both the individual patient and healthcare society in general.

Putting all criteria together, the characteristics of an ideal wound dressing for primary THA/TKA should include: 1) protection against bacterial delivery at the surgical site, 2) maintaining an ideal microenvironment for wound healing while wicking excess exudate from the incision site, 3 ) visually transparent to determine the need for dressing change, 4) ability to adhere to the skin of a moving joint without causing significant skin blistering, and 5) inexpensive and readily available supplies for worldwide use.

At our center we selected the GT dressing as a means to address head-on the competitive field of occlusive postoperative dressings. Our basis for selecting this dressing specifically was multiple. First, Tegaderm is "easy" on the skin. It is thin and mechanically flexible, which is advantageous for application over a moving joint. Our previous experience using Tegaderm over ruptured skin blisters and skin tears showed that it caused minimal marginal dermatitis and blistering. Secondly, the GT dressing construct is a vapor-permeable occlusive film. An important characteristic of Tegaderm is its pore size; the pores are large enough to allow for the exchange of water vapor, but small enough to prevent bacteria from entering into the wound site. The GT dressing keeps the local wound environment moist, preventing excessive drying. Thirdly, the gauze dressing is a highly absorbent material that works on the mechanism of capillary action of its fine threads, effectively wicking fluid from the surgical wound. Furthermore, the white gauze beneath a transparent Tegaderm film allows for the treating physician to easily identify the color and volume of discharge from the wound below. This ease of identification also reduces unnecessary dressing changes. Frequent dressing changes cause episodic cooling of the wound, resulting in a longer time for resuming cellular mitotic activity and, in turn, wound healing [19]. Additionally, each dressing change poses a potential risk of exposing the wound to external nosocomial pathogens. Fourth, the GT dressing provides an essentially waterproof seal. This allows the patient to take a shower the next day postoperatively, if needed. With the skin cleaned and dried in the operating room, we have found that the Tegaderm can stay secure for an extended period of time. We have had patients with an intact GT dressing on the hip and knee for up to 14 days. Fifth, the GT dressing creates a hypoxic environment which has been shown to accelerate angiogenesis [15]. Both moisture and hypoxia are beneficial for wound healing. Lastly, the GT dressing is inexpensive and its supplies are readily available worldwide. At our institution, the cost of a typical GT dressing consisting of $4 \times 4$ gauze sponges and 5 medium-sized Tegaderm films is $\$ 3.00$ USD. A comparable length Aquacel dressing at our institution costs \$37.03 USD.

This review reports a favorable outcome of the majority of primary THA and TKA performed within this study group. We attribute our low overall infection rate to a disci- 
plined comprehensive TJA protocol focusing on minimizing SSI and PJI. Our selection of the GT dressing for postoperative application did not appear to adversely affect our rates of SSI and PJI when compared to other published series [20]. Our low PJI rate is encouraging in light of our series having 49\% B and C grade systemic hosts. The weaknesses of this study are several. First, this was not a randomized trial. Secondly, the total number of subjects studied was relatively small. Per design, we chose first to study the GT dressing construct to see if it was an acceptable dressing for continued use as a perioperative joint dressing for primary THA and TKA. After review of our results, we feel comfortable in stating that the GT dressing meets our criteria as a cost-effective dressing. Going forward, a more rigorous study is needed, At present, we have received IRB approval for a prospective randomized control trial comparing the GT dressing to a proprietary bio-occlusive dressing in primary THA and TKA. The enrollment will exceed 650 primary TJA procedures with a minimum follow-up of 1 year. This RCT will help determine via a rigorous comparison, whether the GT dressing will be equally effective in maintaining a low SSI and PJI rate in primary THA and TKA.

In summary, we introduce the concept of the gauzeTegaderm dressing for use in postoperative primary THA and TKA wounds. This dressing construct meets a majority of criteria to promote wound healing and protect against SSI. The GT dressing has many salutary attributes and our study results show a low rate of SSI and PJI. The GT dressing, thus far, seems to be a reasonable cost-effective dressing that can be utilized worldwide. Our favorable early findings in this review merit a more rigorous investigation of this dressing. An upcoming large volume RCT will delineate the effectiveness of the GT dressing in minimizing postoperative SSI in TJA.

\section{References:}

1. Maradit Kremers H, Larson DR, Crowson CS, Kremers WK, Washington RE, Steiner CA, et al. Prevalence of Total Hip and Knee Replacement in the United States. J Bone Joint Surg Am. 2015;97(17):1386-97.

2. Kurtz SM, Ong KL, Lau E, Widmer M, Maravic M, Gomez-Barrena E, et al. International survey of primary and revision total knee replacement. Int Orthop. 2011;35(12):1783-9.

3. Renaud A, Lavigne M, Vendittoli PA. Periprosthetic joint infections at a teaching hospital in 1990-2007. Can J Surg. 2012;55(6):394-400

4. Greene LR. Guide to the elimination of orthopedic surgery surgical site infections: an executive summary of the Association for Professionals in Infection Control and Epidemiology elimination guide. Am J Infect Control. 2012;40(4):384-6.

5. Arroyo AA, Casanova PL, Soriano JV, Torra IBJE. Open-label clinical trial comparing the clinical and economic effectiveness of using a polyurethane film surgical dressing with gauze surgical dressings in the care of post-operative surgical wounds. Int Wound J. 2015;12(3):285-92.

6. Chowdhry M, Chen AF. Wound dressings for primary and revision total joint arthroplasty. Ann Transl Med. 2015;3(18):268.

7. Hoppenfeld S, de Boer P, Buckley R. Surgical Exposures in Orthopaedics: The Anatomic Approach: Lippincott Williams \& Wilkins; 2016.

8. Ross JA, Greenwood AC, Sasser P, 3rd, Jiranek WA. Periarticular Injections in Knee and Hip Arthroplasty: Where and What to Inject. J Arthroplasty. 2017;32(9S):S77-S80.
9. McPherson EJ DM, Sherif SM. he Fate of the PCL in Cruciate Retaining TKA: A Critical Review of Surgical Technique. Reconstructive Review. . 2013:3(4): 11-7.

10. McPherson EJ, Woodson C, Holtom P, Roidis N, Shufelt C, Patzakis M. Periprosthetic total hip infection: outcomes using a staging system. Clin Orthop Relat Res. 2002(403):8-15.

11. McPherson EJ PC. Musculoskeletal Infection, In: Flynn JM ed. Orthopaedic Knowledge Update 10. Rosemont, IL: American Academy of Orthopaedic Surgeons. 2011:239-58.

12. Parvizi J, Gehrke T, Chen AF. Proceedings of the International Consensus on Periprosthetic Joint Infection. Bone Joint J. 2013;95-B(11):1450-2.

13. Siddique K, Mirza S, Housden P. Effectiveness of hydrocolloid dressing in postoperative hip and knee surgery: literature review and our experience. J Perioper Pract. 2011;21(8):275-8.

14. Falanga V. Occlusive wound dressings. Why, when, which? Arch Dermatol. 1988;124(6):872-7.

15. Ravenscroft MJ, Harker J, Buch KA. A prospective, randomised, controlled trial comparing wound dressings used in hip and knee surgery: Aquacel and Tegaderm versus Cutiplast. Ann R Coll Surg Engl. 2006;88(1):18-22.

16. Sharma G, Lee SW, Atanacio O, Parvizi J, Kim TK. In search of the optimal wound dressing material following total hip and knee arthroplasty: a systematic review and meta-analysis. Int Orthop. 2017;41(7):1295-305.

17. Odland GF. The fine structure of the interrelationship of cells in the human epidermis. J Biophys Biochem Cytol. 1958;4(5):529-38.

18. Kaplan RS, Porter ME. How to solve the cost crisis in health care. Harv Bus Rev. 2011;89(9):46-52, 4, 6-61 passim.

19. Collins A. Does the postoperative dressing regime affect wound healing after hip or knee arthroplasty? J Wound Care. 2011;20(1):11-6.

20. Springer BD, Cahue S, Etkin CD, Lewallen DG, McGrory BJ. Infection burden in total hip and knee arthroplasties: an international registry-based perspective. Arthroplast Today. 2017;3(2):137-40.

\section{SUBMISSION HISTORY}

Submitted December 10, 2017

Reviewed December 15, 2017

Revised December 20, 2017

Accepted December 21, 2017

Published December 31, 2017

AUTHOR AFFILIATIONS

1 Madhav Chowdhry, Matthew Dipane, Edward J. McPherson LA Orthopedic Institute

201 S. Alvarado Street Suite 501, Los Angeles, CA 90057

(Direct inquires to Matthew Dipane, itc@laoi.org)

AUTHOR DISCLOSURES

The authors declare there are no disclosures regarding the publication of this paper.

\section{COPYRIGHT \& OPEN ACCESS}

(C) 2017 Chowdhry, Dipane, McPherson. All rights reserved. Authors retain copyright and grant the journal right of first publication with the work. Reconstructive Review is an open access publication and follows the Creative Commons Attribution-NonCommercial CC BY-NC. This license allows anyone to download works, build upon the material, and share them with others for non-commercial purposes as long as they credit the senior author, Reconstructive Review, and the Joint Implant Surgery \& Research Foundation (JISRF). An example credit would be: "Courtesy of (senior author's name), Reconstructive Review, JISRF, Chagrin Falls, Ohio". 